\title{
Classification of Laser Self-Mixing Interferometric Signal under Moderate Feedback
}

\author{
Olivier D. Bernal, ${ }^{1,2, *}$ Usman Zabit, ${ }^{3}$ and Thierry Bosch ${ }^{1,2}$ \\ ${ }^{1}$ CNRS, LAAS, F-31400 Toulouse, France \\ ${ }^{2}$ Univ de Toulouse, INP, LAAS, F-31400 Toulouse, France \\ ${ }^{3}$ Riphah International University, Islamabad, Pakistan
}

\begin{abstract}
This paper was published in Applied Optics and is made available as an electronic reprint with the permission of OSA. The paper can be found at the following URL on the OSA website: http://dx.doi.org/10.1364/A0.53.000702 Systematic or multiple reproduction or distribution to multiple locations via electronic or other means is prohibited and is subject to penalties under law. (c) 2014 Optical Society of America

This paper presents a different approach to classify Self-Mixing (SM) signals operating in moderate feedback regime. A total of 6 distinct classes of SM signals can be defined based on the SM inherent shapes which depend on both the feedback factor $C$ and the linewidth enhancement factor $\alpha$. This classification allows to clearly identify SM signals for which normalization issues can arise and thus for which displacement precision is inherently reduced due to the very nature of signal itself. Finally, it is shown that phase unwrapping approaches can theoretically retrieve displacement with subnanometer precision for usual laser diodes in moderate feedback regime, in the absence of noise, only for $\alpha$ values greater than 4.3 approximately.
\end{abstract}

OCIS codes: (120.7280) Vibration analysis; (120.3180) Interferometry; (120.0280) Remote sensing and sensors

http://dx.doi.org/10.1364/A0.53.000702 (c) 2014 Optical Society of America

\section{Introduction}

Self-Mixing (SM) interferometry [1-4] remains an active area of research for displacement sensing [58]. For displacement sensing, SM moderate optical feedback regime is being regularly exploited [911] as its supposedly straight-forward signal shape leads to simplified signal processing. The aim of this paper, however, is to demonstrate that 1) SM signals considered to be belonging to moderate feedback regime can be further classified and more importantly 2) certain SM signal classes may not lead to correct displacement retrieval.

SM signal shapes have been reported to depend on the so-called optical feedback coupling factor $C$ as well as the laser diode (LD) linewidth enhancement factor $\alpha$ [1, 2]. Different methods have also been proposed to measure $C$ and $\alpha$ [12 14]. The former parameter $C$, depending notably on the remote target surface reflectivity and the distance

\footnotetext{
* Corresponding author: bernal@enseeiht.fr
}

to the remote target, is usually used to discriminate between three main different optical feedback regimes [1, 2]:

- $0.1<C<1$ : weak feedback regime with sinusoidal or asymmetric SM interferometric fringes devoid of sharp discontinuities.

- $1<C<4.6$ : moderate feedback regime with sawtooth-like SM interferometric fringes exhibiting hysteresis.

- $4.6<C$ : strong feedback regime with fringeloss that ultimately leads to a fringe-less SM signal.

The moderate feedback regime, as already mentioned, is often sought for displacement sensing. Here, displacement can be retrieved either by locking the interferometric phase to half fringe [11] or by unwrapping the interferometric phase. Various SM phase unwrapping methods exist including direct phase unwrapping [15], parametric estimation based unwrapping [16], and phase segmentation based unwrapping [17]. Each of these pro- 
vides increasingly improved displacement measurement precision respectively at a cost of increased processing complexity respectively.

Note that such unwrapping methods seek moderate feedback regime since, in such a regime, SM signals present strong discontinuities which are used by phase unwrapping methods as points of reference 15 18]. Further, a normalization procedure is essentially required by such methods as a first step. However, up to now, to the best of our knowledge, normalization issues were only pointed out for one way target displacement (the target moves in one direction only) [18]. So, in this paper, we shall further analyze in detail moderate regime SM signals in order to know if other kind of normalization issues may exist. It is from this analysis that a novel classification of SM signals in moderate feedback will emerge.

The paper is organized as follows. After a short introduction of the main characteristics of SM signals, we shall derive from established SM equations a new way of classifying SM signals that does not rely only on $C$ value as it has usually been previously done [2], but also takes into account the $\alpha$ value. We shall then be able to show that the moderate feedback regime can be divided into 6 distinct classes out of which only 3 can lead to a proper target displacement retrieval using phase unwrapping methods. Finally, normalization error effects on retrieved displacements will be estimated and a conclusion on the achievable performances of phase unwrapping methods will be drawn.

Let us start with a theoretical background of SM phenomenon.

\section{Theory of Self-Mixing}

The theory of SM interferometry has been described by various authors [19]-20] and is briefly summarized below. Let $D(t)$ represent the instantaneous distance between the LD driven by a constant injection current and a remote surface that backscatters a small amount of optical power back into the LD cavity. To highlight the vibration displacement $d(t), D(t)$ can be expressed as follows:

$$
D(t)=D_{0}+d(t)
$$

where $D_{0}$ is the distance at rest.

When this optical feedback phenomenon occurs, the laser wavelength is no longer the constant $\lambda_{0}$ but is slightly modified and becomes a function of time $\lambda_{F}(t)$ varying with $D(t)$. The wavelength fluctuations can be found by solving the phase equa- tion [2]:

$$
\begin{aligned}
x_{0}(t) & =x_{F}(t)+C \sin \left[x_{F}(t)+\operatorname{atan}(\alpha)\right] \\
& =G\left[x_{F}(t), C, \alpha\right],
\end{aligned}
$$

where $x_{F}$ and $x_{0}$ represent two phase signals (subject to feedback and under free running conditions, respectively).

The value of $x_{F}(t)$ can be extracted from the optical output power (OOP) of the laser diode $P(t)$ using:

$$
P(t)=P_{0}\left\{1+m \cos \left[x_{F}(t)\right]\right\},
$$

where $P_{0}$ is the power emitted by the free running state laser diode and $m$ a modulation index. By using $x_{F}, x_{0}(t)$ can first be retrieved using the nonlinear function $G($ eq 2 ) and then $d(t)$ as shown in [16].

\section{The Proposed SM Classification}

As previously mentioned, three main SM regimes based on $C$ are usually identified. Depending on the SM regime of operation, different algorithms 1517, 21, 22. can be used to unwrap the phase with an aim to achieve either high precision, real-time operation, robustness or a combination of these characteristics. To the best of our knowledge, all the reported unwrapping techniques require one very first step: the normalization. This normalization procedure is based on the assumption that the normalized SM signals always reach the maximum (minimum) value $+1(-1)$ [16]. Though such an assumption is obvious for the weak feedback regime [21, 22], one should evaluate if it is still true for the moderate feedback regime (the strong feedback regime is not considered here due to the higher complexity required to process such SM signals).

Therefore, in this paper, an in-depth study of SM signals in moderate feedback is presented with an aim of determining whether there is a risk of misinterpretation of SM signals during the normalization step. As a result of this analysis, a classification of moderate SM signals for $C>1$ can then be proposed.

To achieve such an objective, it is thus necessary to analyze the evolution of $x_{F}$ around the discontinuities and $G$ as a function of $C$ and $\alpha$.

Firstly, let us analyze $G$ and its nonlinearities.

\section{A. Phase Discontinuity}

$G$ (see eq. 21) is not invertible when $C>1$ [23]. For such values of $C$, the laser diode enters into a multiple mode lasing behavior [20]. This results in phase discontinuities and hysteresis phenomena 


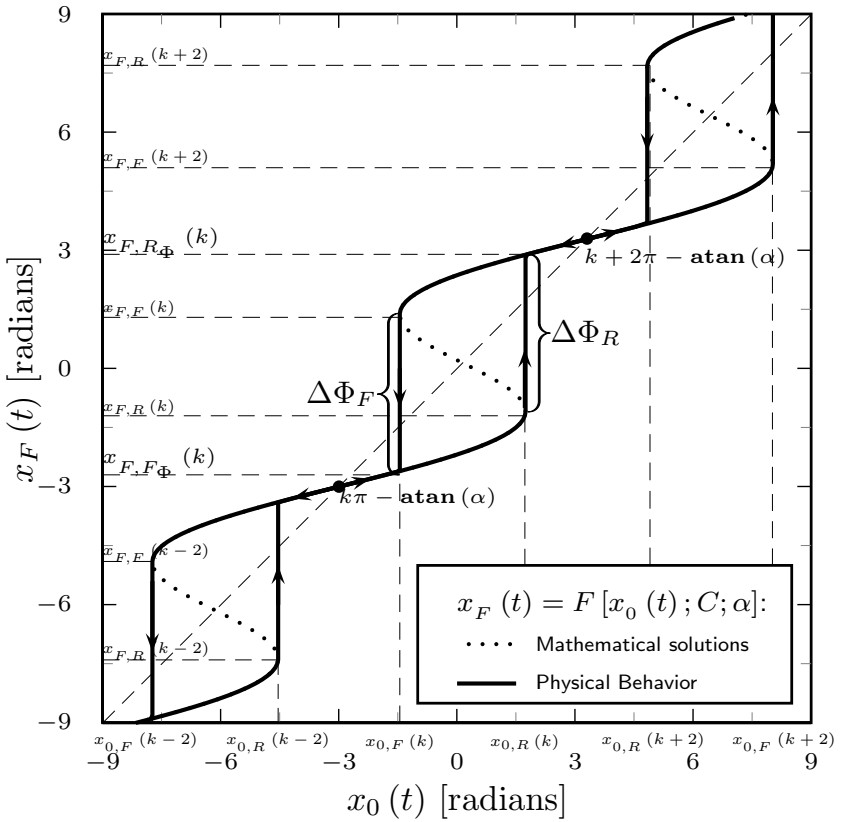

Fig. 1. Transfer function $x_{F}(t)=\mathrm{F}\left[x_{0}(t) ; C, \alpha\right]$ with hysteresis $(C=3$ and $\alpha=2)$ and $k$ is an even integer.

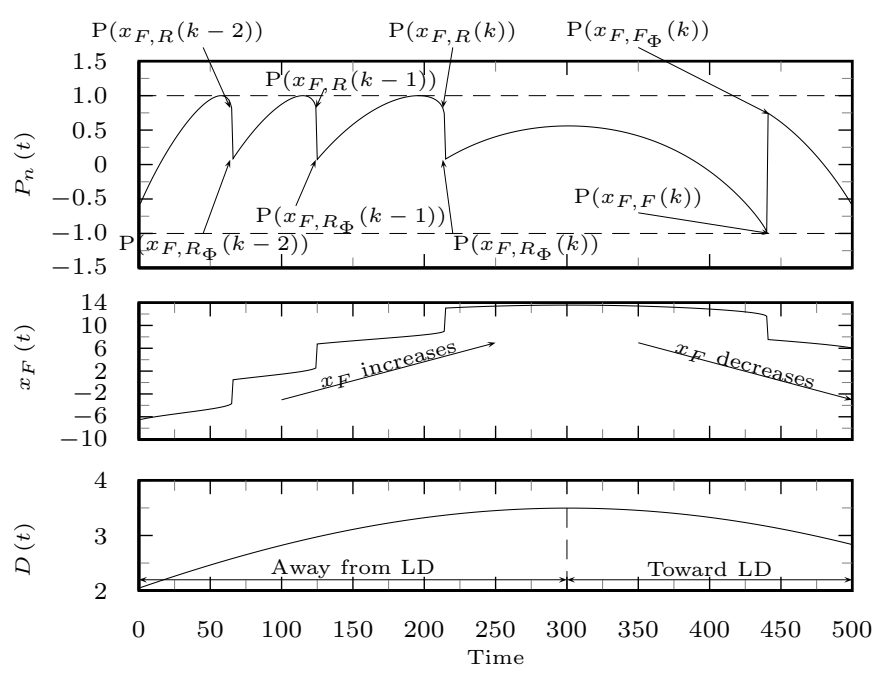

Fig. 2. Graphical representation of the normalized power $P_{n}$ of a SM signal for $C=3$ and $\alpha=2$, its corresponding $x_{F}$ and displacement $D(t)$.

(see Fig. 1). Phase discontinuities occur whenever the function $x_{F}(t)=F\left[x_{0}(t), C, \alpha\right]$ has infinite slopes. These discontinuities can be either rising (subscript R) $\Delta \Phi_{R}$ or falling (subscript F) $\Delta \Phi_{F}$ depending on the evolution of $x_{F}(t)$ (see Fig. 1 and Fig. (2). As these discontinuities strongly affect the LD optical output power (OOP) signals so determining when they occur and what phase shifts are induced in $x_{F}$, is essential to classify SM signals.

From eq2, we can define the following odd func- tion:

$$
H\left[x_{F}, C, \alpha\right]=G\left[x_{F}-\operatorname{atan}(\alpha), C, \alpha\right]-\operatorname{atan}(\alpha),
$$

$G$ presents an inherent symmetry that implies that $\Delta \Phi_{R}=\Delta \Phi_{F}=\Delta \Phi([18])$. Nevertheless, it does not imply that the OOP signal is symmetric. This OOP asymmetry will be studied in detail in the following sections.

The discontinuities $\Delta \Phi_{R}$ occur at some $x_{F, R}$ values obtained for specific $x_{0}$ values: $x_{0, R}$ (see Fig[1).

For $C>1$, at the rising discontinuity, there are at least two possible values for $x_{F}=F\left[x_{0, R}(t), C, \alpha\right]$ : (1) $x_{F, R}$ before the discontinuity and (2) $x_{F, R_{\Phi}}$ after the discontinuity. As a consequence, $\Delta \Phi_{R}$ can be expressed as (see Fig. 1):

$$
\Delta \Phi_{R}=\underbrace{F\left[x_{0, R}(k) ; C ; \alpha\right]}_{x_{F, R_{\Phi}}}-\underbrace{F\left[x_{0, R}(k) ; C ; \alpha\right]}_{x_{F, R}},(
$$

where $k$ is an even integer. In a similar manner, $\Delta \Phi_{F}$ can be expressed as:

$$
\Delta \Phi_{F}=\underbrace{F\left[x_{0, F}(k) ; C ; \alpha\right]}_{x_{F, F_{\Phi}}}-\underbrace{F\left[x_{0, F}(k) ; C ; \alpha\right]}_{x_{F, F}},(6)
$$

However, as the function $\mathrm{G}$ is strongly nonlinear, it is not possible to obtain an exact value of $x_{F, R_{\Phi}}$ $\left(x_{F, F_{\Phi}}\right)$ but only that of $x_{F, R}\left(x_{F, F}\right)$ by equating the derivative of the function $G$ to zero [23]. Such an exact solution is given below as it will be useful for the sake of SM classification:

$$
\begin{aligned}
& x_{F, R}=k \pi-\operatorname{atan}(\alpha)+\beta \\
& x_{F, F}=(k+2) \pi-\operatorname{atan}(\alpha)-\beta,
\end{aligned}
$$

where $k$ is an even integer and $\beta=\operatorname{acos}\left(-\frac{1}{C}\right)$.

Then, by numerically approximating $x_{F, R_{\Phi}}$ and $x_{F, F_{\Phi}}$, Fig 3 (a) and Fig.5 (a) can be obtained. Having known the locations of $x_{F, R}, x_{F, F}, x_{F, R_{\Phi}}$ and $x_{F, F_{\Phi}}$, we shall show how it can be unambiguously determined whether the maximum value +1 or minimum value -1 can be reached. From this last analysis, a new classification of SM signals in moderate feedback will be brought forward and proposed.

\section{B. Classification of SM signals}

By analyzing the value of $x_{F, R}\left(x_{F, F}\right)$ and $x_{F, R_{\Phi}}$ $\left(x_{F, F_{\Phi}}\right)$, it can be determined if individual fringes can reach a maximum value +1 (minimum value 1). This piece of information is essential for the SM normalization which is the first step of any phase unwrapping method algorithms [16, 17].

It is interesting to note that $\left.\operatorname{atan}(\alpha) \in] 0 ; \frac{\pi}{2}\right]$ for $\alpha \in] 0 ; 10]$ and that $\left.\left.\operatorname{acos}\left(\frac{-1}{C}\right) \in\right] \frac{\pi}{2} ; \pi\right]$ for $C \in$ 
a)

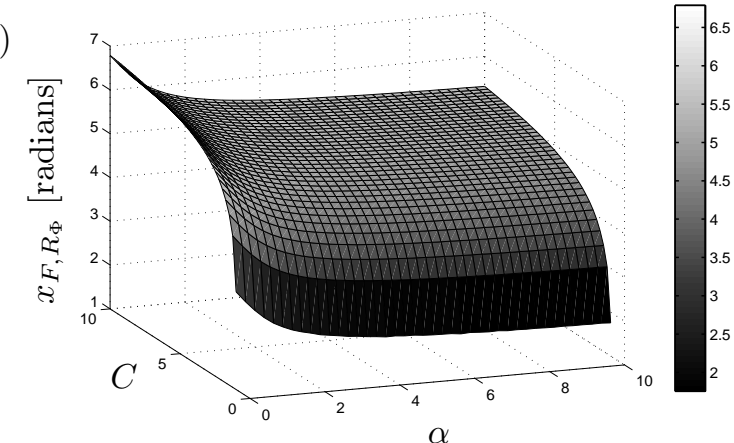

c)

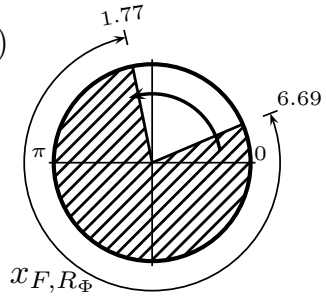

Phase direction of $x_{F}$

Fig. 3. 3D Graphical representation of the phase value $x_{F}$ after the rising discontinuity $x_{F, R_{\Phi}}$ versus $(\mathrm{C} ; \alpha)$ and 2D Graphical representation of the phase value $x_{F}$ before the rising discontinuity $x_{F, R}$ versus $(\mathrm{C} ; \alpha)(\mathrm{a})$ and after (b) the rising discontinuity $x_{F, R_{\Phi}}$ versus $(\mathrm{C} ; \alpha)$ for $(\mathrm{C} ; \alpha) \in(] 1,10] ; 0,10])$.

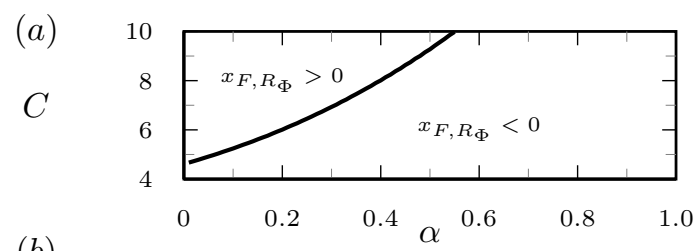

(b)

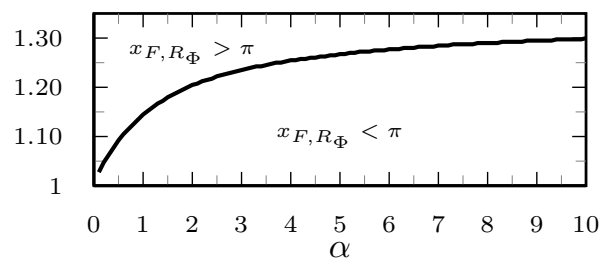

Fig. 4. (a) Contour plot of Fig 3 obtained for $x_{F, R_{\Phi}}=0$ as a function of $(C, \alpha)$ and (b) Contour plot of Fig 3 obtained for $x_{F, R_{\Phi}}=\pi$ as a function of $(C, \alpha)$

]1; 10]. As a result, using eq[7, it can be shown (see Fig 3 (b)) that

$$
\left.\left.\left.x_{F, R}>0[2 \pi] \forall(C, \alpha) \in(11 ; 10] ;\right] 0 ; 10\right]\right),
$$

Therefore, it ensures that the SM signal $\mathrm{R}$ fringes always reach a maximum value +1 before reaching the discontinuity if and only if $x_{F, R_{\Phi}}<0[2 \pi]$ (Fig 3 (c)). Using numerical computation, $x_{F, R_{\Phi}}$ can be evaluated (Fig 3 (a), (c)). As shown in Fig 3 (a) and Fig 4 (a), the case $x_{F, R_{\Phi}}>0[2 \pi]$ happens only a)
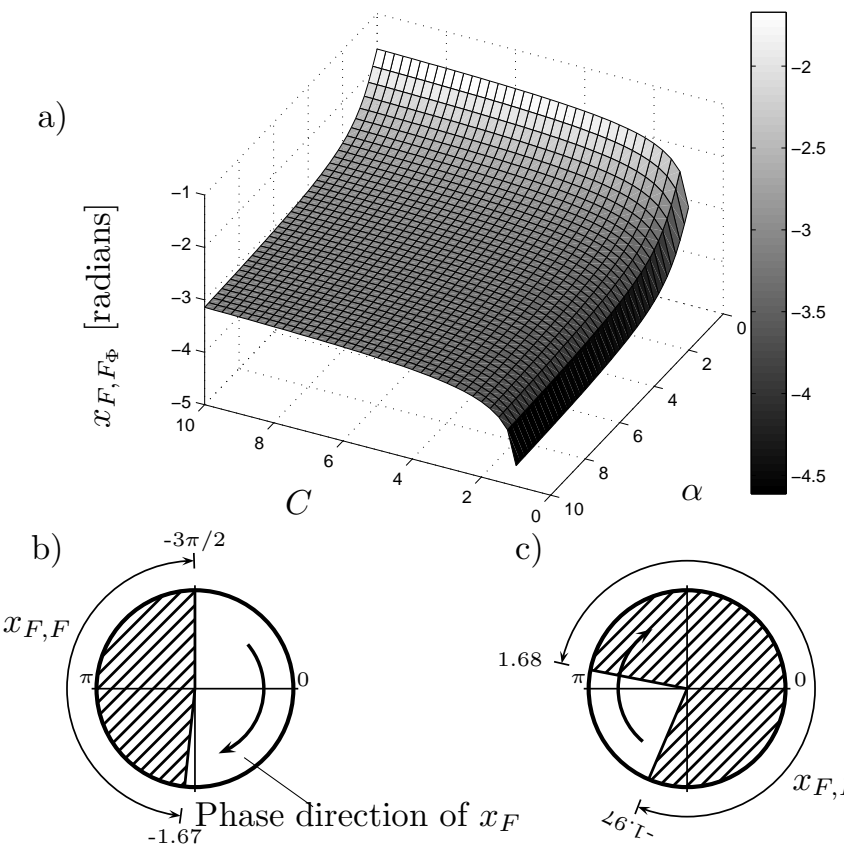

c)

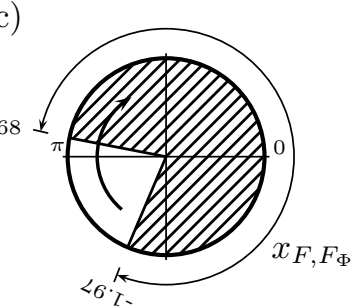

Fig. 5. 3D Graphical representation of the phase value $x_{F}$ after the rising discontinuity $x_{F, F_{\Phi}}$ versus $(\mathrm{C} ; \alpha)$ and $2 \mathrm{D}$ Graphical representation of the phase value $x_{F}$ before the falling discontinuity $x_{F, F}$ versus $(\mathrm{C} ; \alpha)(\mathrm{b})$ and after $(\mathrm{c})$ the falling discontinuity $x_{F, F_{\Phi}} \operatorname{versus}(\mathrm{C} ; \alpha)$ for $(\mathrm{C} ; \alpha) \in(] 1,10] ;] 0,10])$.

for a small set of $(C, \alpha)$ that is out of the moderate feedback regime region. Further, from $x_{F, R_{\Phi}}$, it can also be determined if the fringe can reach a minimum value of -1 . This is only possible if and only if $x_{F, R_{\Phi}}<\pi$ (otherwise -1 is skipped during the discontinuity). As a result, Fig 4 (b) can be numerically obtained.

In a manner similar to that of eq.9 (Fig [5 (b)), it can be deduced that $-\frac{3 \pi}{2}<x_{F, F}<-\frac{\pi}{2}$ for $(C, \alpha) \in$ (] $1 ; 10] ; 10 ; 10])$. This implies that $\mathrm{F}$ fringes might not reach the minimum value of -1 . To ensure this minimum, $x_{F, F}<-\pi[2 \pi]$ should be first verified. It can be shown from eq, that such a condition is valid if:

$$
\left.\left.\alpha>\sqrt{C^{2}-1} \text { for } C \in\right] 1 ; 10\right]
$$

However, as for the $\mathrm{R}$ fringes, it is a necessary condition but it is not sufficient. It should also be checked that $x_{F, F_{\Phi}}<-\frac{3 \pi}{2}$ or that $x_{F, F_{\Phi}}>-\frac{3 \pi}{2}$ and $x_{F, F_{\Phi}}<x_{F, F}$. These conditions can be computationnally tested. Fig 5 (a) is a 3 d plot representing $x_{F, F_{\Phi}}$. As a result, it can be shown that eq.10 (Fig 6) (a)) is necessary and sufficient.

Finally, it can also be determined from $x_{F, F_{\Phi}}$ if the $\mathrm{F}$ fringe can reach a maximum value of 1 . This can easily be checked by numerically solving $x_{F, F_{\Phi}}<0$ (Fig [ (b)). 
(a)

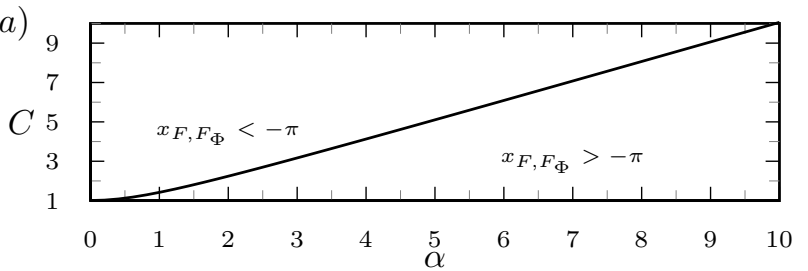

(b)

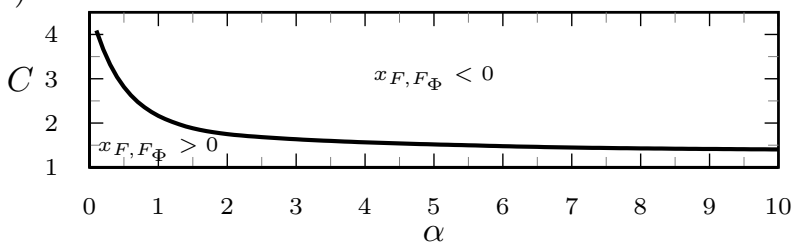

Fig. 6. (a) Graphical solution of $x_{F, F_{\Phi}}=-\pi$ as a function of $(C, \alpha)$; (b) Graphical solution of $x_{F, F_{\Phi}}=0$ as a function of $(C, \alpha)$.

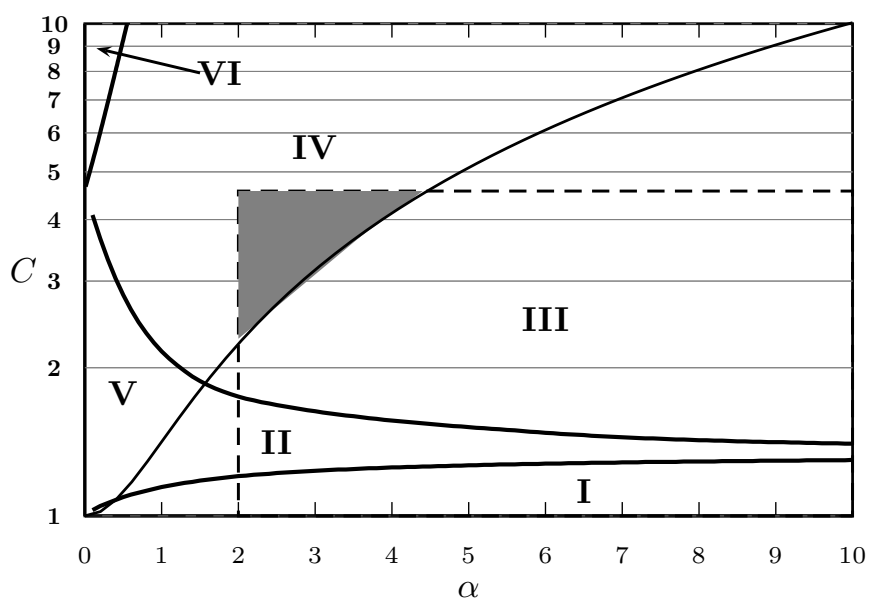

Fig. 7. Graphical representation of the different SM classes as a function of $(C, \alpha)$

This analysis can be summarized by Fig 7 and 8 , It shows that SM signals in moderate regime can be classified into 6 distinct classes depending on values of $C$ and $\alpha$ :

- I: Both the $\mathrm{R}$ and $\mathrm{F}$ fringes reach +1 and -1 .

- II: The $\mathrm{R}$ fringes reach only +1 and the $\mathrm{F}$ fringes can reach both +1 and -1 .

- III: The $\mathrm{R}$ fringes reach only +1 and the $\mathrm{F}$ fringes reach only -1 .

- IV: The $\mathrm{R}$ fringes reach only +1 and the $\mathrm{F}$ fringes do not reach +1 neither -1 .

- $\mathrm{V}$ : Both the $\mathrm{R}$ and $\mathrm{F}$ fringes reach only +1 .

- VI: Both the $\mathrm{R}$ and $\mathrm{F}$ fringes do not reach +1 neither -1.

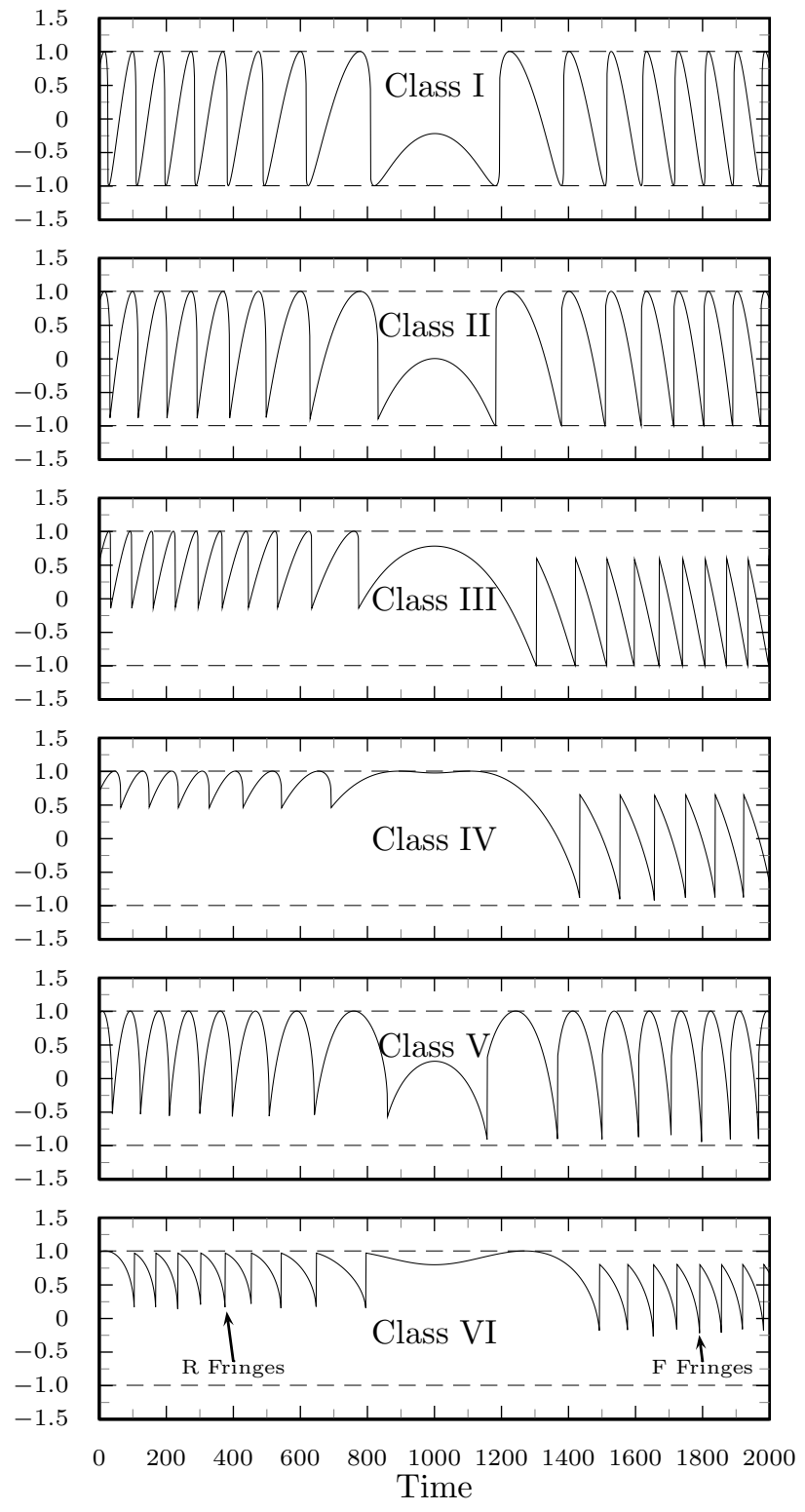

Fig. 8. Graphical representation of SM signals pertaining to the six different classes: (Class I) $C=1.1$ and $\alpha=4$; (Class II) $C=1.3$ and $\alpha=1.5$; (Class III) $C=3$ and $\alpha=4$; (Class IV) $C=4$ and $\alpha=1.5$; (Class V) $C=1.3$ and $\alpha=0.5$; (Class VI) $C=9$ and $\alpha=0.2$.

Contrary to the SM signals belonging to the classes I to IV, the classes V and VI can only be obtained for low linewidth enhancement factor (LEF). As shown in 24] 25], quantum cascade laser can achieve such low LEF values. Though SM signals (similar to class $\mathrm{V}$ signal) can be obtained using such laser sources (as seen in Fig. 3 (b) of [25]), SM signals are usually obtained by using laser diodes with a LEF greater than 2 (as tabulated in [13]). 
Consequently, the classes of interest are mostly the classes I, II, III and IV which correspond to usually encountered SM signals (see Fig, 8 ). In Fig:7, the dashed rectangle represents the usual $(C, \alpha)$ set of experimental interest. It is of upmost importance to note that it is possible to normalize without any ambiguity only the SM signals of classes I, II, and III. Further, the shapes of class III and class IV SM signals are very similar. Therefore, performing the correct normalization on IV SM signal is very challenging and can induce reconstruction displacement errors. We propose to analyze this error to assess whether or not it can compromise the results obtained by phase unwrapping approach if the SM class recognition is not properly done.

\section{Classification and Phase Unwrapping}

Let's consider a IV SM signal as shown in Fig. 8 to illustrate the error analysis. Its misinterpretation as a class III SM signal results in stretching the SM signals and thus, in wrongly extracting the phase $x_{F}$ from the OOP using the arccosine function (eq. 3). Due to the behavior of this arccosine function, this phase estimation is maximum for OOP value close to 1 and -1 . Further, we do know that the minimum value of OOP is achieved at $x_{F, F}$ (eq 8). Consequently, the $x_{F}$ phase estimation error is maximum for this value. If the estimation error on the estimation of $C$ and $\alpha$ is not considered, then an estimation of the phase error $\delta x_{0}$ around $x_{F, F}$ can be expressed as follows:

$$
\begin{aligned}
\delta x_{0}= & -\delta x_{F, F}\left(1+C \cos \left(x_{F, F}+\operatorname{atan}(\alpha)\right)\right) \\
& -\frac{\left(\delta x_{F, F}\right)^{2}}{2} C \sin \left(x_{F, F}+\operatorname{atan}(\alpha)\right),
\end{aligned}
$$

where

$$
\delta x_{F, F}=\pi-\operatorname{atan}(\alpha)-\operatorname{acos}\left(-\frac{1}{C}\right),
$$

From Fig 9 , it is clear that the lower the $\alpha$ value, the bigger the phase estimation error. For example, for $\alpha=2$ and $C=4$, the estimated phase error is approximately $0.086 \mathrm{rad}$ which induces a displacement estimation error of $5.5 \mathrm{~nm}$ for a $800 \mathrm{~nm}$ wavelength laser.

Further, in order to accurately reconstruct the displacement, phase unwrapping approaches need to use the $C$ and $\alpha$ value [17, 18]. In [18], a joint estimation of $C$ and $\alpha$ is employed. However for class IV signals, using such an algorithm, $C$ is underestimated because of the normalization that induces a reduction of the phase discontinuity $\Delta \Phi$ (Fig(1). This affects directly the estimated displacement (eq.2).

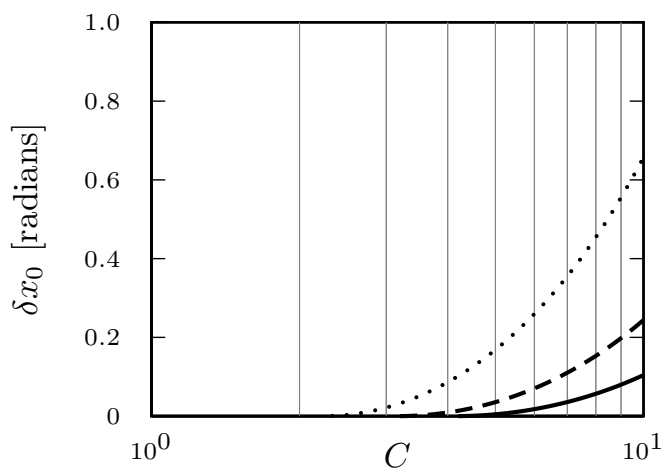

Fig. 9. Graphical representation of the phase error $\delta x_{0}$ as a function of $C$ for $\alpha=2$ (dotted), $\alpha=3$ (dashed), $\alpha=4$ (plain).
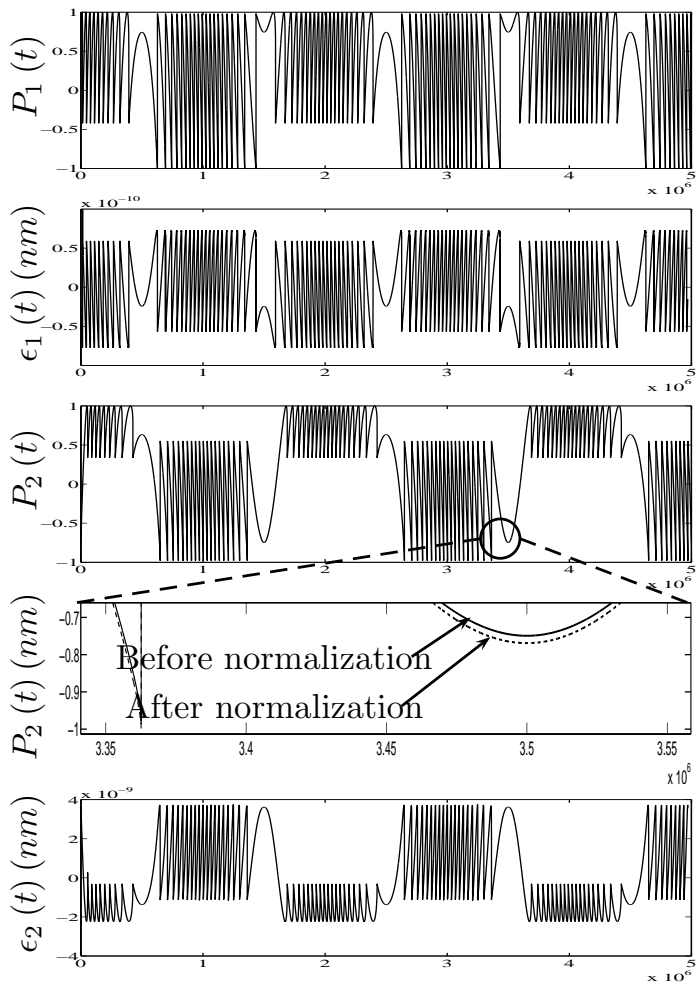

Fig. 10. Simulated SM signals $P_{1}(t)$ of class III (a) and $P_{2}(t)$ of class IV (c) and their corresponding displacement reconstruction errors $\epsilon_{1}(t)$ (b) and $\epsilon_{2}(t)$ (e) for $(C, \alpha)=(2,2)$ and $(C, \alpha)=(4,2)$ respectively. For $\lambda_{0}=800 \mathrm{~nm}$ and a sinusoidal displacement amplitude of $4 \mu \mathrm{m}$.

To illustrate this analysis, Fig 10 shows two simulated SM signals (III and IV classes) and their reconstructed displacement error using the phase unwrapping method described in 18]. The maximum reconstruction error is approximately $4 \mathrm{~nm}$ for 
the class IV (Fig 10 (d)) compared to $70 \mathrm{pm}$ for the class III (Fig $10(\mathrm{~b}))$. Further, the estimated C values are 2.0007 instead of 2 and 3.91 instead of 4 for the class III and IV respectively. These results are in accordance with the theoretical analysis. However, the estimated errors are still lower than the phase unwrapping method reconstruction errors for measured SM signals (approximately $40 \mathrm{~nm}$ for 16. and $14 \mathrm{~nm}$ for [18]). Therefore, as shown in Fig. 7. using a laser diode having an $\alpha$ value greater than 4.3 is a necessary condition to achieve subnanometer precision for any SM signal processing methods requiring SM signal normalization as a first step.

\section{Conclusion}

In this paper, a new SM signal classification has been proposed which is based on the characteristic shapes of the SM signals as a function of $C$ and $\alpha$. It has been shown that out of the 6 different classes of moderate regime SM signals, only 3 can lead to an exact and proper displacement reconstruction due to normalization issues. Due to the relatively low values required of $\alpha$, class $\mathrm{V}$ and VI are unlikely to be encountered for most laser diodes. The displacement error for the class IV has been estimated in particular as such a SM signal can be misinterpreted as class III SM signal which does not suffer from normalization errors. The inherent estimated error of phase unwrapping methods due to a lack of discrimination between class III and class IV SM signals is approximately $<10 \mathrm{~nm}(C \in[1,4]$, $\alpha \in[2,10]$ and $\left.\lambda_{0}=800 \mathrm{~nm}\right)$. Finally, it is shown that phase unwrapping approaches can theoretically retrieve displacement with subnanometer precision for usual laser diodes in moderate feedback regime, in the absence of noise, only for $\alpha$ values greater than 4.3 approximately.

\section{Acknowledgment}

Finally, this work might not have been possible had it not been for the funding of the French ANR Emergence.

\section{References}

[1] S. Donati, "Developing self-mixing interferometry for instrumentation and measurements," Laser \& Photon. Rev., vol. 6, p. 393417, 2012.

[2] G. Giuliani and S. Donati, Unlocking Dynamical Diversity - optical feedback effects on semiconductors lasers, D. M. Cane and K. A. Shore, Eds. John Wiley \& Sons, Ltd, 2005.

[3] R. Teysseyre, F. Bony, J. Perchoux, and T. Bosch, "Laser dynamics in sawtooth-like self-mixing signals," Opt. Lett., vol. 37, no. 18, pp. 3771-3773, 2012.
[4] S. Donati and M. Norgia, "Self-mixing interferometry for biomedical signals sensing," Selected Topics in Quantum Electronics, IEEE Journal of, vol. 20, no. 2, pp. 6900108-6900108, 2014.

[5] U. Zabit, O. Bernal, and T. Bosch, "Self-mixing laser sensor for large displacements: Signal recovery in the presence of speckle," Sensors Journal, IEEE, vol. 13, no. 2, pp. 824-831, 2013.

[6] A. Magnani, A. Pesatori, and M. Norgia, "Selfmixing vibrometer with real-time digital signal elaboration," Appl. Opt., vol. 51, no. 21, pp. 5318-5325, 2012.

[7] Y. Leng Lim, P. Dean, M. Nikolic, R. Kliese, S. P. Khanna, M. Lachab, A. Valavanis, D. Indjin, Z. Ikonic, P. Harrison, E. H. Linfield, A. Giles Davies, S. J. Wilson, and A. D. Rakic, "Demonstration of a self-mixing displacement sensor based on terahertz quantum cascade lasers," $A p$ plied Physics Letters, vol. 99, no. 8, pp. 081108081 108-3, 2011.

[8] L. Lu, W. Zhang, B. Yang, J. Zhou, H. Gui, and B. Yu, "Dual-channel self-mixing vibration measurement system in a linear cavity fiber laser," Sensors Journal, IEEE, vol. 13, no. 11, pp. 4387-4392, 2013.

[9] R. Atashkhooei, J.-C. Urresty, S. Royo, J.-R. Riba, and L. Romeral, "Runout tracking in electric motors using self-mixing interferometry," Mechatronics, IEEE/ASME Transactions on, vol. PP, no. 99, pp. 1-7, 2012.

[10] U. Zabit, O. Bernal, and T. Bosch, "Design and analysis of an embedded accelerometer coupled selfmixing laser displacement sensor," Sensors Journal, IEEE, vol. 13, no. 6, pp. 2200-2207, 2013.

[11] G. Martini, E. Randone, and S. Donati, "Very low frequency self-mixing laser diode vibrometer," in Sensors, 2012 IEEE, 2012, pp. 1-4.

[12] J. Xi, Y. Yu, J. Chicharo, and T. Bosch, "Estimating the parameters of semiconductor lasers based on weak optical feedback self-mixing interferometry," Quantum Electronics, IEEE Journal of, vol. 41, no. 8, pp. 1058-1064, 2005.

[13] Y. Yu, G. Giuliani, and S. Donati, "Measurement of the linewidth enhancement factor of semiconductor lasers based on the optical feedback self-mixing effect," Photonics Technology Letters, IEEE, vol. 16, no. 4, pp. 990-992, 2004.

[14] Wen Zhao, Huiying Ye, and Yanguang Yu, "Design of Measurement Algorithm of Feedback Strength Factor of Optical Feedback Self-mixing Interferometry Systems based on System Generator," Proceedings of the 2nd International Conference on Computer Science and Electronics Engineering, doi:10.2991/iccsee.2013.182, 2013.

[15] M. Norgia and A. Pesatori, "Fully analog selfmixing laser vibrometer," in Instrumentation and 
This paper was published in Applied Optics and is made available as an electronic reprint with the permission of OSA. The paper can be found at the following URL on the OSA website: http://dx.doi.org/10.1364/A0.53.000702 Systematic or multiple reproduction or distribution to multiple locations via electronic or other means is prohibited and is subject to penalties under law. (c) 2014 Optical Society of America

Measurement Technology Conference (I2MTC), 2011 IEEE, 2011, pp. 1-4.

[16] C. Bes, G. Plantier, and T. Bosch, "Displacement measurements using a self-mixing laser diode under moderate feedback," Instrumentation and Measurement, IEEE Transactions on, vol. 55, no. 4, pp. $1101-1105$, aug. 2006.

[17] Y. Fan, Y. Yu, J. Xi, and J. F. Chicharo, "Improving the measurement performance for a self-mixing interferometry-based displacement sensing system," Applied Optics, vol. 50, no. 26, pp. 5064-5072, 2011.

[18] O.D. Bernal, U. Zabit, and T. Bosch, "Study of laser feedback phase under self-mixing leading to improved phase unwrapping for vibration sensing," Sensors Journal, IEEE, vol. 13, no. 12, pp. 49624971, 2013.

[19] R. Lang and K. Kobayashi, "External optical feedback effects on semiconductor injection laser properties," IEEE J. Quantum Electron., vol. 16, no. 3, pp. 347-355, 1980.

[20] K. Petermann, "External optical feedback phenomena in semiconductor lasers," IEEE J. Sel. Topics Quantum Electron., vol. 1, no. 2, pp. 480-489, 1995.
[21] C. Bes, V. Belloeil, G. Plantier, Y. Gourinat, and T. Bosch, "A self-mixing laser sensor design with an extended kalman filter for optimal online structural analysis and damping evaluation," Mechatronics, IEEE/ASME Transactions on, vol. 12, no. 3, pp. 387-394, 2007.

[22] A. Doncescu, C. Bes, and T. Bosch, "Displacement estimation with an optical feedback interferometer using an evolutionary algorithm," in Sensors, 2007 IEEE, 2007, pp. 382-386.

[23] G. Plantier, C. Bes, and T. Bosch, "Behavioral model of a self-mixing laser diode sensor," IEEE J. Quantum Electron., vol. 41, no. 9, pp. 1157-1167, 2005.

[24] J. von Staden, T. Gensty, and W. Elser, "Measurements of the $\alpha$ factor of a distributed-feedback quantum cascade laser by an optical feedback selfmixing technique," Opt. Lett., vol. 31, no. 17, pp. 2574-2576, 2006.

[25] N. Kumzaki, Y. Takagi, M. Ishihara, K. Kasahara, N. Akikusa, and T. Edamura, "First direct observation of small linewidth enhancement factor of fabryperot quantum cascade laser," Japanese Journal of Applied Physics, vol. 47, no. 3, pp. 1606-1608, 2008. 